\title{
The Relationship between ABO Blood Group Type and the COVID-19 Susceptibility in Qatif Central Hospital, Eastern Province, Saudi Arabia: A Retrospective Cohort Study
}

\author{
Ghada Ali Aljanobi, Alya Hassan Alhajjaj, \\ Fatimah Lateef Alkhabbaz, Jumana Mansour Al-Jishi \\ Internal Medicine Department, Qatif Central Hospital, Qatif, KSA \\ Email: dr.ghadaaljanobi@gmail.com
}

How to cite this paper: Aljanobi, G.A., Alhajjaj, A.H., Alkhabbaz, F.L. and Al-Jishi, J.M. (2020) The Relationship between ABO Blood Group Type and the COVID-19 Susceptibility in Qatif Central Hospital, Eastern Province, Saudi Arabia: A Retrospective Cohort Study. Open Journal of Internal Medicine, 10, 232-238.

https://doi.org/10.4236/ojim.2020.102024

Received: May 14, 2020

Accepted: June 6, 2020

Published: June 10, 2020

Copyright $\odot 2020$ by author(s) and Scientific Research Publishing Inc. This work is licensed under the Creative Commons Attribution International License (CC BY 4.0).

http://creativecommons.org/licenses/by/4.0/

\section{(c) (i) Open Access}

\begin{abstract}
Introduction: It has been found that the viral infection is linked to the $\mathrm{ABO}$ blood group. Limited studies investigated the relationship of the ABO blood group and susceptibility to COVID-19 infection. Objective: To investigate the association between the ABO blood group and the COVID-19 susceptibility. Material \& Methods: The study is a retrospective cohort study. It included all hospitalized confirmed COVID-19 patients in Qatif central hospital, Eastern province, Saudi Arabia. We included both genders and people who are older than 14 years of age. The study was conducted in the period between $15^{\text {th }}$ March, and $15^{\text {th }}$ April, 2020. Results: Our sample consists of 72 patients; we found a percentage distribution of $23.62 \%, 33.33 \%, 9.72 \%$, and $33.33 \%$ for blood groups $\mathrm{A}, \mathrm{B}, \mathrm{AB}$ and $\mathrm{O}$, respectively. Our results showed significantly increased risk for $A B$ blood groups $(P<0.05)$ and significantly lower risk for the $\mathrm{O}$ blood group $(\mathrm{P}<0.05)$. Although there was a higher percentage distribution of the B blood group among COVID-19 patients as compared to the blood donors' groups, this difference did not reach statistical significance. There was no significant difference in the risk of COVID-19 in blood group A with a distribution of $23.60 \%$ in blood donors' group, and $23.62 \%$ in COVID-19 patients. Conclusion: Our findings suggest that among confirmed COVID-19 patients, patients with $\mathrm{AB}$ blood group have higher susceptibility while patients with $\mathrm{O}$ blood group have lower susceptibility to COVID-19 infection. Our study has limitations and further studies are needed.
\end{abstract}

\section{Keywords}

ABO Group, Saudi, Qatif, COVID-19 


\section{Introduction}

The novel coronavirus SARS-CoV-2, which triggers the current COVID-19 infectious disease, is rapidly spreading across the world today [1].

Owing to the rapid dissemination of COVID-19, there has been a dramatic rise in the number of patients affected and 3,267,184 patients have been diagnosed, with 229,971 deaths to our present time. In Saudi Arabia, up to the present time, there are 24,097 patients, with 169 deaths [2].

Current clinical findings indicate that the age, gender and comorbidity of patients are the key risk factors predicting the extent of COVID-19 infection [1].

No biological markers have been identified so far for predicting COVID-19 susceptibility. Although blood group types are genetically inherited, environmental factors may theoretically influence the next generations' ABO blood group type in a population [3].

It has been found that viral infections are linked to the $\mathrm{ABO}$ blood group. ABO blood group antigens may affect the vulnerability to norwalk virus infection [4]. One study also found that blood group $\mathrm{O}$ has lower susceptibility to hepatitis B, C, HIV, syphilis or malaria [5]. Cheng et al. had predicted the susceptibility of SARS-CoV infection in Hong Kong from the ABO blood group systems. The authors found that the hospital staff with blood group $\mathrm{O}$ had a lower risk of being infected as compared to those with non-O blood group [6].

So far, there are only two studies that investigated the relationship of ABO blood group with susceptibility to COVID-19 infection. The first study was conducted in Wuhan city, China [3]. The second study was done in New York, United States [7].

Here, we investigated the relationship between the ABO blood group type and the susceptibility to COVID-19 in patients who were admitted at Qatif Central Hospital (QCH).

\section{Methods}

\subsection{Study Design and Participants}

Retrospective cohort analysis, it included all hospitalized confirmed COVID-19 patients in QCH, eastern province, Saudi Arabia. It included both genders and who are older than 14 years of age. The study was conducted in the period between March 15, and April 15, 2020.

\subsection{Data Collection}

The confirmed COVID-19 patients' medical records were reviewed by the trained research team of the medical department, QCH.

Covid-19 was diagnosed based on provisional guidelines from the WHO. A confirmed case of Covid-19 was identified as a positive result for nasal and pharyngeal swab specimens in real-time reverse transcriptase-polymerase-chain-reaction (RT-PCR) assay [8]. ABO blood group from all patients was analyzed with column agglutination method. 
Blood bank registry at QCH was reviewed for the blood donors for the year 2019 to estimate the distribution of ABO blood groups. Those data were used for comparison with the $\mathrm{ABO}$ blood groups of our patients.

SOFA (Sequential Organ Failure Assessment) score was used to assess the severity of the illness in critical care patients. SOFA is based on six different variables, one for each system (respiratory, cardiovascular, renal, hepatic, neurological systems, and coagulation). Each system scored between 0 and 4, and higher scores are associated with worse organ dysfunction and prognosis [9].

\subsection{Statistical Analysis}

Statistical analysis was performed using IBM, SPSS version 26. Standard descriptive and analytics statistics will be used to analyze the data. Chi-square test was used to test for significant difference and $\mathrm{P}$-value $\leq 0.05$ will be considered significant.

\subsection{Ethic Consideration}

The study was voluntary and informed consent was taken from all participants. The study was approved by the institutional research ethic committee of Qatif central hospital, Saudi Arabia (QCH-SREC0197/2020).

\section{Results}

The ABO blood groups of 5291 blood donors in Qatif central hospital, Qatif city, Eastern province showed a percentage distribution of $23.60 \%, 23.87 \%, 3.91 \%$ and $48.62 \%$ for blood groups $\mathrm{A}, \mathrm{B}, \mathrm{AB}$ and $\mathrm{O}$ respectively [10].

We studied hospitalized COVID-19 positive patients in QCH. Our sample consists of 82 patients but $\mathrm{ABO}$ blood group data was available for 72 patients only (Table 1).

We found a percentage distribution of $23.62 \%, 33.33 \%, 9.72 \%$ and $33.33 \%$ for blood groups $\mathrm{A}, \mathrm{B}, \mathrm{AB}$ and $\mathrm{O}$ respectively. It was found that the proportion of $\mathrm{AB}$ blood group was significantly higher in patients with COVID-19 compared to the blood donors $(9.72 \%$ vs $3.91 \%)$ with significant probability $(\mathrm{P}<0.05)$. The proportion of O blood group was found to be lower in COVID-19 patients as compared to blood donors (33.33\% vs $48.62 \%$ ), which was statistically significant $(\mathrm{P}<0.05)$. These results corresponded with significantly increased risk for COVID-19 for AB blood groups with an OR of 2.645 (95\% CI 1.198 - 5.839) and significantly lower risk for O blood group with an OR of 0.529 (95\% CI 0.32 0.87). Although there was higher percentage distribution of B blood group among COVID-19 patients as compared to the blood donors, this difference did not reach statistical significance. There was no significant difference for risk of COVID-19 in blood group A with distribution of $23.60 \%$ in the blood donors, and $23.62 \%$ in COVID-19 patients (Table 2).

A different distribution of blood groups was found among patients with critical conditions. In our sample of COVID-19 patients, four patients were admitted to the ICU, two of which has A blood group and the other two have O blood 
group. The Sequential Organ Failure Assessment (SOFA) score for them revealed higher scores for A blood groups patients. However, these findings were not statistically significant (Table 3 ).

Moreover, we investigated $\mathrm{ABO}$ blood groups distribution in regards to sex and age groups distribution. Our study had a percentage distribution of males and females of $33.3 \%$ and $66.7 \%$ respectively (Table 1 ). There was no relationship between gender, age and the $\mathrm{ABO}$ blood group (Table 4, Table 5).

Table 1. Characteristics of the patients.

\begin{tabular}{cc}
\hline Characteristics & All Patients ( $\mathbf{n}=\mathbf{7 2})$ \\
\hline Median age (IQR)-yr & $50(36-58)$ \\
Male-No. (\%) & $24(33.3)$ \\
Female-No. (\%) & $48(66.7)$ \\
Chronic illnesses-No. (\%) & $37(51.4)$ \\
Contact-No. (\%) & $26(36.1)$ \\
Travel-No. (\%) & $37(51.4)$ \\
\hline
\end{tabular}

Table 2. ABO risk assessment.

\begin{tabular}{cccc}
\hline ABO system & SARS-CoV2 Infected $(\mathrm{n}=72)$ & OR $(95 \% \mathrm{CI})$ & P Value $^{*}$ \\
\hline A-No. (\%) & $17(23.6)$ & $1.000(0.58-1.73)$ & 0.999 \\
B-No. (\%) & $24(33.3)$ & $1.595(0.97-2.61)$ & 0.062 \\
AB-No. (\%) & $7(9.7)$ & $2.645(1.20-5.84)$ & 0.024 \\
O-No. (\%) & $24(33.3)$ & $0.529(0.32-0.87)$ & 0.010 \\
\hline
\end{tabular}

Abbreviations: CI, confidence interval; OR, odds ratio; SARS-CoV2, severe acute respiratory syndrome coronavirus 2 . ${ }^{*}$ v value was calculated by 2 -tailed $\chi^{2}$ comparing each group individually to the combined other groups.

Table 3. Critical patients' characteristics.

\begin{tabular}{ccccc}
\hline Total patient $(\mathrm{n}=4)$ & Blood group & SOFA score & Outcome & P value \\
\hline Patient No. 1 & A & 14 & Recovered & 0.235 \\
Patient No. 2 & O & 2 & Recovered & 0.597 \\
Patient No. 3 & O & 5 & Recovered & 0.597 \\
Patient No. 4 & A & 16 & Recovered & 0.235 \\
\hline
\end{tabular}

Abbreviations: SOFA, sequential organ failure assessment.

Table 4. Blood groups distribution in different age groups.

\begin{tabular}{ccccccc}
\hline Blood group & $\begin{array}{c}\mathbf{1 5 - 2 9} \\
(\mathbf{n}=10)\end{array}$ & $\begin{array}{c}\mathbf{3 0 - 4 4} \\
(\mathbf{n}=21)\end{array}$ & $\begin{array}{c}45-59 \\
(\mathbf{n}=27)\end{array}$ & $\begin{array}{c}\mathbf{6 0 - 7 4} \\
(\mathbf{n}=13)\end{array}$ & $\begin{array}{c}\geq 75 \\
(\mathbf{n}=1)\end{array}$ & P value \\
\hline A-No. (\%) & $2(20.0)$ & $7(33.3)$ & $5(18.5)$ & $2(15.4)$ & $1(100)$ & 0.403 \\
B-No. (\%) & $2(20.0)$ & $8(38.1)$ & $10(37.0)$ & $4(30.8)$ & $0(0)$ & 0.862 \\
AB-No. (\%) & $0(0)$ & $2(9.6)$ & $2(7.5)$ & $3(23.0)$ & $0(0)$ & 0.447 \\
O-No. (\%) & $6(60.0)$ & $4(19.0)$ & $10(37.0)$ & $4(30.8)$ & $0(0)$ & 0.610 \\
\hline
\end{tabular}


Table 5. Blood groups distribution in different genders.

\begin{tabular}{cccc}
\hline Blood group & Male $(\mathbf{n}=\mathbf{2 4})$ & Female $(\mathbf{n}=\mathbf{4 8})$ & P value \\
\hline A-No. (\%) & $8(33.3)$ & $9(18.8)$ & 0.239 \\
B-No. (\%) & $8(33.3)$ & $16(33.3)$ & 1.000 \\
AB-No. (\%) & $1(4.2)$ & $6(12.5)$ & 0.412 \\
O-No. (\%) & $7(29.2)$ & $17(35.4)$ & 0.791 \\
\hline
\end{tabular}

\section{Discussion}

Several studies investigated the relationship between the ABO blood groups and different viral infections. To the best of our knowledge, this is the first study in the Middle East and the third study in the world that demonstrates the relationship between ABO blood groups and COVID-19 infection. The first study was conducted in China and it was published March, 2020 [3]. The second study was conducted in New York, USA, and published on April, 2020 [7].

In our COVID-19 patients, we found that blood groups $\mathrm{B}$ and $\mathrm{O}$ were the most frequent blood groups between patients (33.33\%). On the other hand, the most frequent blood group among 5291 blood donors for the year 2019 was blood group $\mathrm{O}$ followed by $\mathrm{AB}$ blood group.

According to different studies done in the Eastern province, Saudi Arabia, ABO blood groups distribution was different. A study was conducted by Mohieldin Elsayid et al. to estimate the prevalence of Saudi patients' blood group in King Abdulaziz Medical City, Riyadh. It showed that blood group $\mathrm{O}$ was the most frequent blood group in the Saudi population, while blood group AB was the lowest [11]. The variability in the frequency of blood groups and Rh phenotypes is due to variations in ethnic and geographical disparities as well as movement of migration [11]. On the other hand, Bashawri et al. concluded that O-positive blood group was the most common blood group in the Saudi population while blood group A was held at lower frequencies [12].

Comparing the ABO blood groups in our COVID-19 patients with the blood groups in our donors, we found that there was significantly increased risk for COVID-19 in patients with AB blood groups and significantly lower risk for patients with O blood groups. Our finding was different from the Chinese study which showed that blood group A was associated with an increased risk for COVID-19 infection. On the other hand, our study was similar to their study that blood group O was associated with a lower risk for COVID-19 infection [3].

The mechanisms causing certain blood groups predisposition for corona virus infection are not clear, though there are several unproved theories including anti blood group antibodies [13].

In our sample, there was no clear correlation between the $\mathrm{ABO}$ blood groups and COVID-19 infection severity, most likely because the number of our critical patients was small.

Our study has several limitations: 
First, sample size was small (72 patients) which is related to the number of hospitalized infected COVID-19 patients in QCH. Second, number of severe cases was very limited (4 patients) with no mortality case. Third, multivariate regression analysis couldn't be done because of deficient information regarding the control group (blood donors).

\section{Conclusion}

Our finding suggests that among confirmed COVID-19 patients, patients with AB blood group had high susceptibility while patients with O blood group had low susceptibility to COVID-19 infection. Due to the above limitations, we could not conclude that there was a relationship between $\mathrm{ABO}$ blood groups and COVID-19 infection susceptibility. Further studies are needed with large sample size and control groups.

\section{Acknowledgements}

Dr. Sameera Alsafawni for her help to access the ABO blood group for the blood donors. Dr. Suad Albeesh for literature review and to all health providers during COVID-19 pandemics.

\section{Conflicts of Interest}

The authors declare no conflicts of interest regarding the publication of this paper.

\section{References}

[1] Chen, N., Zhou, M., Dong, X., Qu, J., Gong, F., Han, Y., Yu, T., et al. (2020) Epidemiological and Clinical Characteristics of 99 Cases of 2019 Novel Coronavirus Pneumonia in Wuhan, China: A Descriptive Study. The Lancet, 395, 507-513. https://doi.org/10.1016/S0140-6736(20)30211-7

[2] World Health Organization (2020) Coronavirus Disease 2019 (COVID-19): Situation Report.

https://www.who.int/emergencies/diseases/novel-coronavirus-2019/situation-reports

[3] Zhao, J., Yang, Y., Huang, H.P., Li, D., Gu, D.F., Lu, X.F., Zhang, Z., Liu, L., Liu, T., Liu, Y.K. and He, Y.J. (2020) Relationship between the ABO Blood Group and the COVID-19 Susceptibility. https://doi.org/10.1101/2020.03.11.20031096

[4] Lindesmith, L., Moe, C., Marionneau, S., Ruvoen, N., Jiang, X.I., Lindblad, L., Baric, R., et al. (2003) Human Susceptibility and Resistance to Norwalk Virus Infection. Nature Medicine, 9, 548-553. https://doi.org/10.1038/nm860

[5] Batool, Z., Durrani, S.H. and Tariq, S. (2017) Association of ABO and Rh Blood Group Types to Hepatitis B, Hepatitis C, HIV and Syphillis Infection, a Five Year' Experience in Healthy Blood Donors in a Tertiary Care Hospital. Journal of Ayub Medical College Abbottabad, 29, 90-92.

http://jamc.ayubmed.edu.pk/index.php/jamc/article/view/1729/874

[6] Cheng, Y., Cheng, G., Chui, C.H., Lau, F.Y., Chan, P.K., Ng, M.H., Wong, R.S., et al. (2005) ABO Blood Group and Susceptibility to Severe Acute Respiratory Syndrome. JAMA, 293, 1447-1451. https://doi.org/10.1001/jama.293.12.1450-c 
[7] Zietz, M. and Tatonetti, N.P. (2020) Testing the Association between Blood Type and COVID-19 Infection, Intubation, and Death. https://doi.org/10.1101/2020.04.08.20058073

[8] Tahamtan, A. and Ardebili, A. (2020) Real-Time RT-PCR in COVID-19 Detection: Issues Affecting the Results. Expert Review of Molecular Diagnostics, 20, 453-454. https://doi.org/10.1080/14737159.2020.1757437

[9] Lambden, S., Laterre, P.F., Levy, M.M. and Francois, B. (2019) The SOFA ScoreDevelopment, Utility and Challenges of Accurate Assessment in Clinical Trials. Critical Care, 23, Article No. 374. https://doi.org/10.1186/s13054-019-2663-7

[10] Al Qatif (2019) Qatif Central Hospital. Blood Group Analysis. Blood Bank.

[11] Elsayid, M., Aseeri, Y.Y., Saqri, F.A., Alanazi, A. and Qureshi, S. (2015) A Study of Prevalence of Blood Group of Saudi Patients in King Abdulaziz Medical City-Riyadh. Science Journal of Public Health, 3, 559-562. https://doi.org/10.11648/j.sjph.20150304.25

[12] Bashwari, L.A., Al-Mulhim, A.A., Ahmad, M.S. and Ahmed, M.A. (2001) Frequency of ABO Blood Groups in the Eastern Region of Saudi Arabia. Saudi Medical Journal, 22, 1008-1012. https://www.ncbi.nlm.nih.gov/pubmed/11744976

[13] Guillon, P., Clément, M., Sébille, V., Rivain, J.G., Chou, C.F., Ruvoën-Clouet, N. and Le Pendu, J. (2008) Inhibition of the Interaction between the SARS-CoV Spike Protein and Its Cellular Receptor by Anti-Histo-Blood Group Antibodies. Glycobiology, 18, 1085-1093. https://doi.org/10.1093/glycob/cwn093 